\title{
Detection of gene expression changes at chromosomal rearrangement breakpoints in evolution
}

\author{
Adriana Muñoz ${ }^{1,2^{*}}$, David Sankoff ${ }^{2}$ \\ From ACM Conference on Bioinformatics, Computational Biology and Biomedicine 2011 (ACM-BCB) \\ Chicago, IL, USA. 1-3 August 2011
}

\begin{abstract}
Background: We study the relation between genome rearrangements, breakpoints and gene expression. Genome rearrangement research has been concerned with the creation of breakpoints and their position in the chromosome, but the functional consequences of individual breakpoints remain virtually unknown, and there are no direct genome-wide studies of breakpoints from this point of view. A question arises of what the biological consequences of breakpoint creation are, rather than just their structural aspects. The question is whether proximity to the site of a breakpoint event changes the activity of a gene.
\end{abstract}

Results: We investigate this by comparing the distribution of distances to the nearest breakpoint of genes that are differentially expressed with the distribution of the same distances for the entire gene complement. We study this in data on whole blood tissue in human versus macaque, and in cerebral cortex tissue in human versus chimpanzee. We find in both data sets that the distribution of distances to the nearest breakpoint of "changed expression genes" differs little from this distance calculated for the rest of the gene complement. In focusing on the changed expression genes closest to the breakpoints, however, we discover that several of these have previously been implicated in the literature as being connected to the evolutionary divergence of humans from other primates.

Conclusions: We conjecture that chromosomal rearrangements occasionally interrupt the regulatory configurations of genes close to the breakpoint, leading to changes in expression.

\section{Background}

The phenotypic consequences of genome rearrangements in humans, such as infertility or developmental pathologies when these mutations occur in the germ line, and cancer when they occur in somatic cells, are well documented [1] and often understood down to the level of changes in gene expression. The classic example is the Philadelphia $\mathrm{t}(9 ; 22)(\mathrm{q} 34 ; \mathrm{q} 11)$ translocation creating the Philadelphia chromosome [2] and the BCR-Abl fusion gene whose tyrosine kinase product has wideranging molecular interactions ultimately responsible for

\footnotetext{
* Correspondence: adri.embo@gmail.com

'School of Information Technology \& Engineering, University of Ottawa,

Ottawa, K1N 6N5, Canada

Full list of author information is available at the end of the article
}

chronic myeloid leukemia. The situation with the homozygotic rearranged genomes of reproductively isolated populations is quite different. The breakpoints of the evolutionary rearrangements differentiating these genomes are known to co-occur with a large number of genomic features, such as regions that are gene-rich regions, GC-rich, hypomethylated, duplicated, pericentromeric or subtelomeric, as often reviewed (e.g., [3]), but the functional consequences of individual breakpoints remain virtually unknown, and there are few direct genome-wide studies of breakpoints from this point of view. An early comparison of the chimpanzee and human genomes [4] found genes on rearranged chromosomes tended to change expression, and included a report that some, unspecified, genes within $2 \mathrm{Mb}$ of 
breakpoints, or in the same chromosomal band region, changed more than others, but this work was limited by the small set of breakpoints only known at that time from cytogenetic studies in the early 1980s [5].

In this paper, we propose a new paradigm for this type of investigation. The idea is basically to compare any changes of expression of genes that are close to, or even disrupted by, chromosomal breakpoints in the comparison of two genomes with changes affecting the gene complement more generally, controlled of course for tissue and experimental conditions. This is not a trivial exercise. There are now high-resolution techniques to identify breakpoint regions [6-8], and thousands of data sets containing the results of whole-genome microarray assays, but comparative, whole genome data sets, controlled for tissue, with orthologous chromosomal positions specified for two species, are not easy to come by [9].

We have been able to make use of two, relatively early, tissue-controlled comparisons of orthologs in humans and non-human primates, the first [10] on whole blood tissue in macaques and humans, and the second [11] on the cerebral cortex of chimpanzees and humans. The blood comparison lacks chromosomal positioning of genes, and does not examine chromosomal rearrangements. The cerebral cortex study relies on breakpoint data from early cytological studies only. Both suffer, for our purposes, from obsolete gene nomenclature. Although we have implemented a system for high throughput analysis, the largely manual conversion of gene names remains a bottleneck that will only be relaxed when more comparative expression data becomes available using current gene and marker terms.

In the next section, we first formalize the null hypothesis of no systematic relationship between gene expression and proximity to breakpoints. We then describe the ortholog expression data sets, the breakpoint data sets, and our protocol for linking the two, as well as the details of our method and its implementation. In the following section, we present the statistical results of our study on change of expression near breakpoints. We find little evidence for rejecting the null hypothesis in either the human-macaque whole blood tissue data set or the human-chimpanzee cerebral cortex dataset. For the few genes closest to breakpoints that do change expression, however, several have previously been tied to have some interesting correlates. Then, in the Conclusions, we discuss the potential for larger scale studies within this paradigm.

\section{Results}

\section{The null hypothesis}

Were there no association between breakpoint creation and change of expression of neighbouring genes, we would expect changed-expression genes to be spatially distributed independently of breakpoint positions.
Consider the interval determined by the position $a_{1}$ and $a_{2}$ of the two breakpoints on either side of a changedexpression gene. Let $u=\left|a_{1}-a_{2}\right| / 2$. The position of the gene, considered as a random variable $y$ should be uniformly distributed in the interval $\left[y_{\min }, y_{\min }+2 u\right]$ where $y_{\text {min }}=\min \left(a_{1}, a_{2}\right)$. The distance $x$ to the closest breakpoint will then be distributed as a uniform variable on the interval $[0, u]$.

For visualization purposes, since the scale of intergenic distances is of the order of hundredths or thousandths of inter-breakpoint distances, we will study the distribution of $z=\log x$ rather than of $x$. Since $x$ is uniform on $[1, u]$, the probability density of $z$ will have the form of a truncated positive exponential distribution

$$
p(z)=e^{z-u},
$$

for $0 \leq z \leq u$, as in Figure 1a.

Since the distance $2 u$ between the breakpoints will itself be distributed randomly (as the distance between two order statistics, namely a negative exponential) and depend on the length of the chromosome and the number of breakpoints, the empirical distribution of distances is predicted by a sum of variables, all with density $p(z)$ but with different parameters $u$, as in Figure $1 \mathrm{~b}$.

\section{The data}

To assess this hypothesis, we need to choose two genomes that are closely enough related that a comparison of gene expression still carries a signal of events in their recent evolutionary divergence, but distant enough so that there are numerous rearrangement breakpoints in their genomic alignment. For our purposes, we should also have relations of orthology established across the two genomes. Of the many expression databases available, there are few that satisfy these criteria. In the future, however, we can expect many more evolutionoriented genome-wide expression projects and this motivates our preliminary study. The present study is confined to two comparisons, one of the human and macaque genomes and gene expression in whole blood samples, and the second of human and chimp genomes and gene expression in cerebral cortex tissue. The tools we use to analyze these data, however, are applicable to much wider datasets.

\section{The gene expression data}

The gene expression data: whole blood tissue Dillman et al. [10] analyzed whole blood tissue in human and three closely related non-human primates (NHP) namely the rhesus macaque, the cynomologous macaque, and the green african monkey. Each of their probe sets was defined by 54,000 probes, representing 38,500 genes from the completely sequenced human genome (2004 release). 

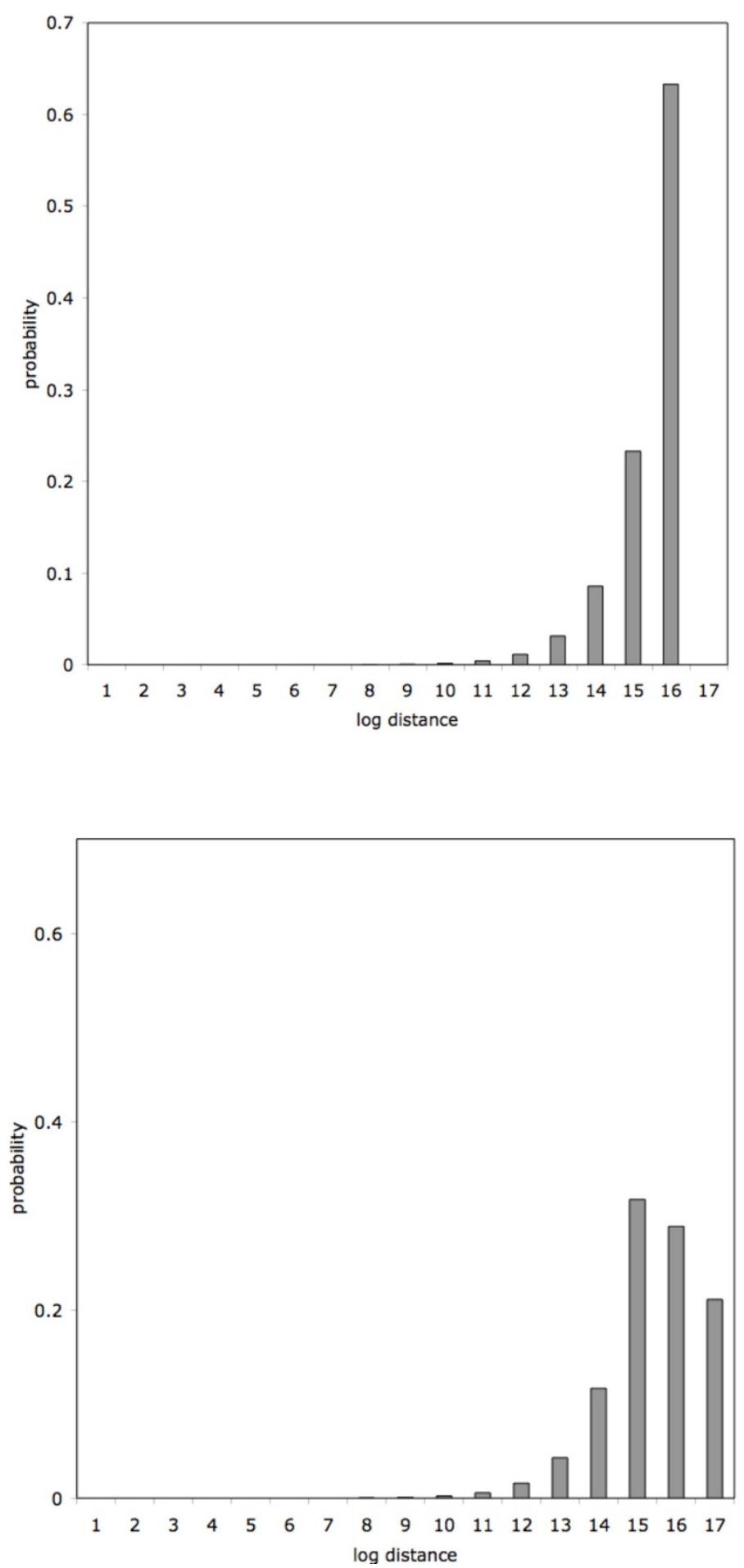

Figure 1 Null hypotheses. a) Distribution of $z=\log$ distance to nearest breakpoint, under the null hypothesis, with $u=e^{16}$. b) Predicted empirical frequency distribution, based on equally weighted $u=15,16,17$.

The gene expression profiles for non-human primates (NHP) and human whole blood tissue were compared using a variety of statistical techniques (principal components, hierarchical clustering, analysis of variance) in order to find genes differentially expressed in humans and NHPs. The results include genetic elements identified as genes, mRNAs and ESTs. 
Note that where these data tell us a gene is expressed more in one genome than the other, it does not tell us whether expression increased in the first genome since divergence from a common ancestor, or whether it decreased in the other.

We extracted 317 genetic elements with significant fold change from this table to use in testing our hypotheses. It is important to note that there is no gene coordinate or BPR information in the gene expression database. Thus, the crux of our investigation is to relate these unpositioned expression data associated with gene names to the breakpoint data, which is simply positional, with no gene names. To do so, we require a database containing both name and positions of human genes.

The gene expression data: cerebral cortex tissue The second case study is confined to the comparison of the chimpanzee and human genomes and gene expression in brain tissue. It is based on a separate study by Cáceres et al. [11] where they analyzed brain tissue in human, chimpanzee and rhesus macaque using rhesus macaque as an outgroup.

Cáceres et al. [11] analyzed brain tissue in human, chimpanzee and rhesus macaque using rhesus macaque as an outgroup. They measured gene expression levels by using Affymetrix human microarrays. Each of their probe sets was defined by 12,625 probes, representing 10,000 genes.

We loaded the set of 80 differentially expressed genes that were up-regulated or down-regulated in chimp into the gene expression database as described in the preceding Section.

\section{The breakpoint data}

Breakpoints can today be determined more precisely than with the classical cytogenetics methods [6]. Comparing different human genomes, the position of the breakpoint can by determined down to the nucleotide level [12], but this is not generally for inter-specific comparisons where positional homology may not be well-defined especially for non-coding regions [13]. Lemaitre et al. [7] compared the genomes of human and five mammals: dog, mouse, rat, macaque and chimp, using a methodology that allowed them to delineate evolutionary breakpoint regions along the human genome with a finer resolution than observed previously.

These authors defined a breakpoint region $(B P R)$ in the human genome as "a region that underwent at least one large chromosomal structural change, or is orthologous to such region in a non-human lineage".

They performed pairwise comparisons between human and the other mammals and identified 622 non-intersecting BPRs ranging from 1 to 2,887,673 nucleotides with a mean size of $104 \mathrm{~kb}$. Those 622 BPRs are stored in a database of sets of coordinates of breakpoints, organized by chromosome, in the format of Table 1 .

Breakpoints for the whole blood tissue study To compare the macaque genome to the human, we extracted only those breakpoints, 92 of them, on evolutionary branches leading to these species from their most recent common ancestor, namely those labelled in the dataset as human, human-chimp or macaque, as illustrated in Figure 2. All other breakpoints are found in both human and macaque or in neither.

It is important to note that there is no systematic accounting of gene expression or even of gene information in the BPR database, although these features of the human genome (but not in other genomes) played a role in the characterization of BPR regions in [7].

Breakpoints for the cerebral cortex study There are too few breakpoints on the human genome during the period of evolutionary divergence from chimpanzee to be able to carry out our study, and the breakpoints in [7] are only given in terms of the human genome.

Thus, we identified chimpanzee-human orthologs using Biomart [14], and we ran the Cassis software [8] to identify 38 breakpoints on the chimp genome during the period of evolutionary divergence from humans. We then loaded this set of breakpoints into the breakpoint database as described in the preceding Section.

\section{The genome database}

Since the breakpoint data are stored in UCSC Genome Browser [15] coordinates, we used the entire set of human genes from the human genome assembly of May, 2004 (NCBI35 or hg17) from this browser as a baseline against which to test our differentially expressed genes in human and macaque whole blood tissue. This set is more comprehensive and more accurately positioned than the original set of "no changed expression" genes in the original two studies.

\section{Table 1 Database of BPRs}

\begin{tabular}{lccr}
\hline Chromosome & Begin & End & Evolutionary branch \\
\hline chr1 & 10382322 & 10382387 & dog \\
chr1 & 109923784 & 109923788 & chimp \\
chr1 & 143495190 & 143766399 & macaque \\
chr1 & 144691208 & 144707142 & primates \\
chr1 & 144850157 & 145574145 & chimp \\
chr1 & 150079680 & 150138541 & dog \\
chr3 & 126855424 & 127207816 & primates \\
chr3 & 128287101 & 128299344 & macaque \\
chr5 & 102756311 & 102787215 & mouse \\
chr5 & 110090786 & 110287080 & rodents \\
chr5 & 112304457 & 112304458 & rat \\
chr22 & 34277622 & 34286037 & radents \\
chr22 & 37056914 & 37068605 &
\end{tabular}




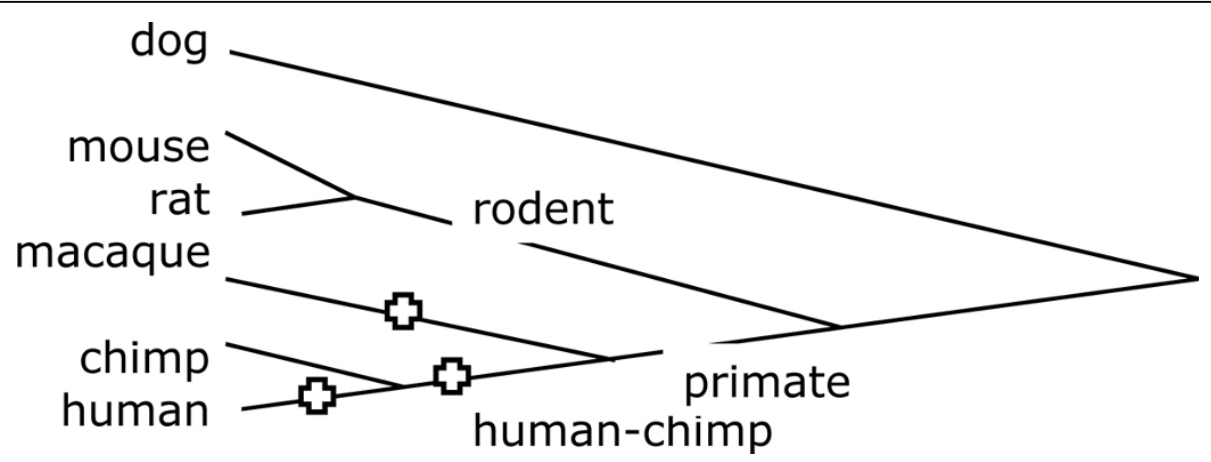

Figure 2 Phylogenetic relationships of species. Phylogeny of species in the breakpoint database, with branches pertinent to the humanmacaque comparison indicated.

For our cerebral cortex study, we used the entire set of chimpanzee genes from the chimpanzee genome assembly of March, 2006 (CHIMP2.1) and the human genome assembly of February, 2009 (NCBI37 or GRCh37) from the Ensembl Genome Browser [16] as a basis for comparison of our differentially expressed genes.

\section{Making connections}

We first sketch the general protocol for linking each breakpoint dataset with the corresponding expression data set via the UCSC gene browser. We then describe how we implemented this in a way that can handle data sets much larger than those available for the present study. As:

1. quantitative measures of gene expression become more accurate,

2. as gene terminology become standardized across genomes,

3. as data on multiple tissues are generated, and

4. as we compare more highly rearranged genomes,

it will be useful to have a high throughput system to generate the data for statistical analysis.

Link breakpoints and expression via gene names The protocol is as follows.

1. Scan the gene expression database for genes showing significant fold change in the human-macaque or human-chimpanzee comparison and extract the human gene name.

2. Locate the records for these differentially expressed genes in the genome browser, by matching names in the two databases. This step is not fully automated since a good proportion of the "names" in the whole blood tissue expression database are not gene names at all, but are ESTs or transcripts of part of the gene, which can be located in other UCSC browser files, or obsolete gene names, which have to be tracked down by web search. A full 50 of the 317 differentially expressed elements in the human-macaque study did not have hits at all in the UCSC browser, and had to be dropped from our analysis. Eight of the 80 differentially expressed genes in the cerebral cortex study were discarded for the same reason.

3. Extract the chromosome and coordinates of these genes in the human genome, and in the case of the cerebral cortex study, in the chimpanzee genome.

4. Compute the distance in nucleotides to the closest $B P R$ in the BPR database.

5. Similarly, for all the human genes in the full genome browser that do not match those differentially expressed genes previously identified, compute the distance in nucleotides to the closest BPR.

Having extracted all these data on 267 differentially expressed genes from the whole blood tissue expression database, or the 72 differentially expressed genes from the cerebral cortex expression database, as well as the corresponding information on the rest of the human gene complement, we are now in a position to treat them statistically.

Implementation We designed a relational database schema, implemented in PostgreSQL [17], to integrate the three different kinds of dataset: BPRs, differentially expressed genetic elements and all human genes. We loaded this with the data described in the preceding Breakpoint data, Gene expression data and Genome database sections. We also loaded UCSC browser counterparts of the mRNA and EST entries found in the differentially expressed genes database. In addition, we loaded the entire set of human genes from the UCSC Known Genes Table. For the cerebral cortex study, we loaded the entire set of chimpanzee genes from the chimpanzee genome assembly of March, 2006 (CHIMP2.1) and the human genome assembly of February, 2009 (NCBI37 or GRCh37) from the Ensembl Genome Browser [16] into the genome database. 
We queried the relational database with a series of SQL statements implementing the different steps described in the preceding Section in order to link the information and compute the distance $d$ between each differentially expressed gene and its closest BPR, as well as the distance between each gene in the remainder of the human gene complement, and its closest BPR.

\section{Results of analyses Whole blood tissue}

Figure 3 (top) compares the distance to the nearest breakpoint of differentially expressed genes to that of the entire set of human genes located in all of the chromosomes that contains breakpoints. While the shape of the distribution is generally as expected from our model illustrated in Figure 1, it is clear that there is little difference between the distributions for the differentially expressed genes and the rest of the human gene complement. This is what we would expect if rearrangement generally has no impact on gene expression. However, this does not mean that rearrangement never has this effect.

This prompted us to inspect more closely the small number of differentially expressed genes close to BPRs for each chromosome that contains breakpoints: one in chromosome 16 where $d=216$ and four in chromosomes 1,2 and $\mathrm{X}$ with $d<10^{5}$. As a visualization tool, our distribution on a log scale depicts this neatly, as in Figure 4. for genes in chromosome 16 and chromosome 1.

\section{NBPF}

Though the few differentially expressed genes close to breakpoints that we found do not seem to be functionally related, it is of interest that one of those on human chromosome 1 is member 10 of the Neuroblastoma breakpoint family (NBPF). This family was so named because of a patient with a constitutional translocation $t$ $(1 ; 17)(\mathrm{p} 36 ; \mathrm{q} 12-21)$ breakpoint near a gene family member, thought to suppress formation of this tumour, eventually developed a neuroblastoma [18]. This gene family is known to evolve rapidly in the primates, by full and partial duplication and divergence [19], has undergone a rapid recent expansion reflected in copy number variation in humans [20], and is thought to play a role in the physiological divergence of primate species. Thus it is of particular interest that one family member near an evolutionary breakpoint has changed expression level in the whole blood tissue study.

\section{Cerebral cortex study}

As in the blood study, Figure 3 (bottom) compares the distance to the nearest breakpoint of differentially expressed genes to that of the entire set of chimpanzee genes located in one of the chromosomes that contains breakpoints, in the cerebral cortex data. Again the shape of the distribution is as expected from our model illustrated in Figure 1, so that there is little difference between the distributions for the differentially expressed genes and the rest of the human gene complement.

Again, however, we examined a number of differentially expressed genes close to BPRs in chimpanzee for each chromosome that contains breakpoints: one in chromosome 1 where $d=9.5 \mathrm{Kbp}$ and four in chromosomes 19, 15, and 17 where $d$ between $d=2.2$ and $d=$ 3.2 Mbp. As a visualization tool, our distribution on a $\log$ scale depicts this neatly, as in Figure 5 for genes in chromosome 1 and chromosome 19 in the chimpanzee genome. It is interesting to note the two closest differentially expressed genes to a BPR in chromosome 19 shared the same BPR where $d=2.2$ and $d=2.6 \mathrm{Mbp}$, respectively (see Figure 5, bottom).

\section{MAPT}

The MAPT gene was the closest differentially expressed gene to a breakpoint in chromosome 17 in the chimpanzee vs. human comparison, where $d=3.2 \mathrm{Mbp}$. This gene codes for Tau, a protein involved in the nucleation, elongation, and stabilization of microtubules [21]. It is associated with a group of human neurodegenerative diseases characterized by the presence of filamentous Tau deposits in nerve cells and glial cells $[21,22]$, such as Alzheimer's disease (AD), progressive supranuclear palsy (PSP), and frontotemporal dementia and parkinsonism linked to chromosome 17(FTDP-17)). The chimpanzee brain has a relative resistance to developing Tau pathology [23]. Since humans and great apes have very similar Tau protein sequences, differences in intronic sequence might explain their differential susceptibility to developing filamentous Tau inclusions, in particular, the apparent resistance of the chimpanzee to developing a filamentous Tau pathology in the brain [24]. The proximity of the MAPT gene to an evolutionary breakpoint that we have pointed out here, in connection with its changed expression level in chimpanzee brain tissue, suggests that the wider chromosomal environment of the gene may also play a role in the resistance of the chimpanzee to developing Tau pathologies.

\section{The retinoblastoma genes}

The Retinoblastoma 1 gene $R B 1$ on chromosome 13 regulates cell growth and proliferation in the brain and other organs, and the suppression of both copies of this gene is associated with an embryonic neoplasm of retinal origin called retinoblastoma. A study of the high degree of sequence conservation of RB1 in human and primates supports a hypothesis of purifying selection in $R B 1$ throughout the history of primates [25].

We found that the Retinoblastoma-like 2 (RBL2) gene was the closest differentially expressed gene to a breakpoint in chromosome 16 in the chimpanzee vs. human 

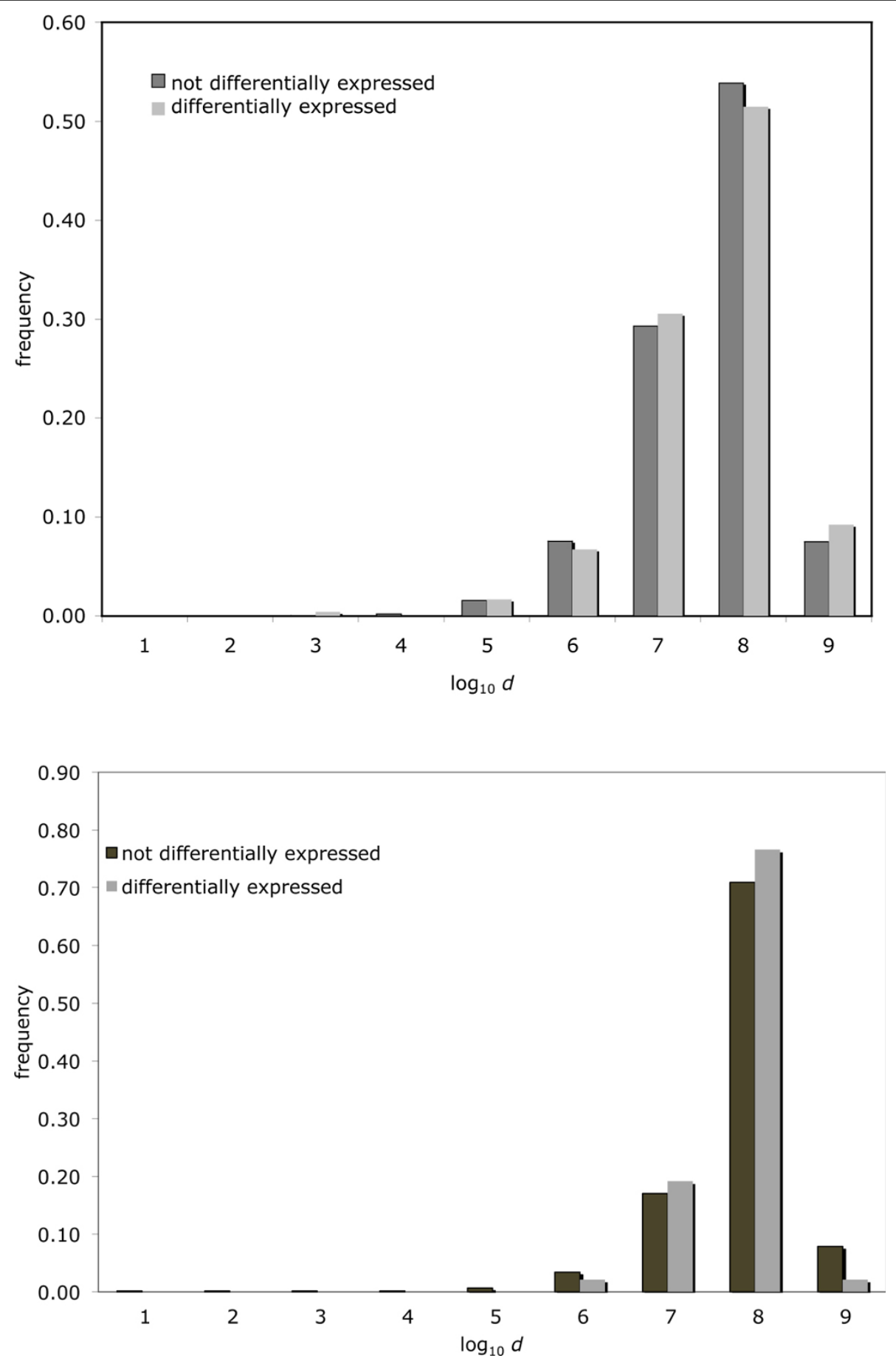

Figure 3 Differential expression and distance to breakpoint. Histogram of distances from genes to closest BPR for differentially expressed genes vs. not differentially expressed genes for all chromosomes. Top: whole blood tissue. Bottom: cerebral cortex tissue.

comparison, where $d=7 \mathrm{Mbp}$. This gene, down-regulated in chimpanzee, regulates $R B 1$.

In addition, in our survey, we found that the retinoblastoma-binding protein 5 (RBBP-5) was the closest differentially expressed (up-regulated) gene to a breakpoint in chromosome 1 in the same comparison, where $d=$ $9.5 \mathrm{Kbp}$.

The facts that both $R B L 2$ and $R B B P-5$ interact with the highly conserved $R B 1$, and that both change expression consequent to rearrangement events, suggest a 

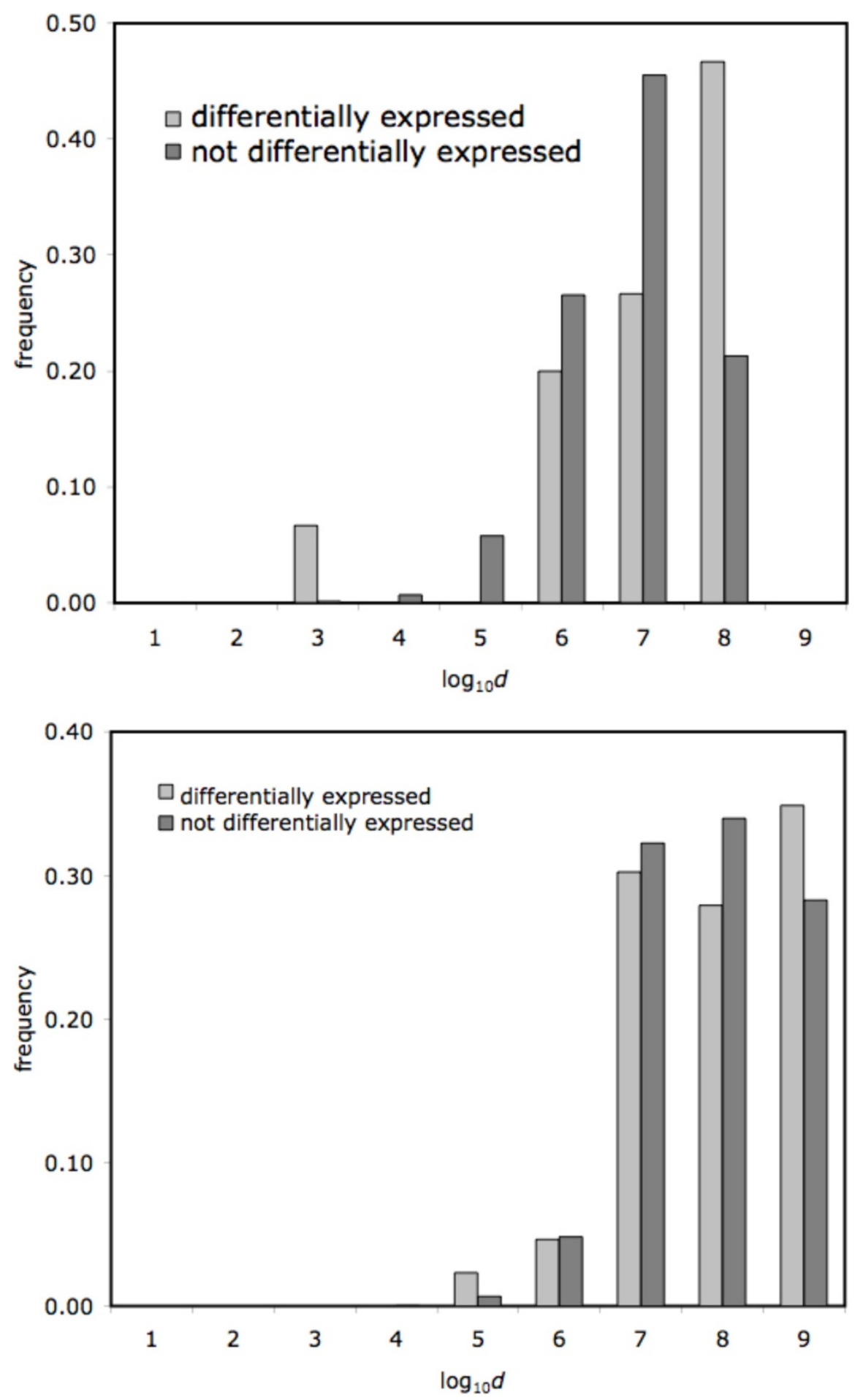

Figure 4 Expression in blood and distance to breakpoint for two chromosomes. Histogram of distances from genes to closest BPR for differentially expressed genes vs. not differentially expressed genes for chromosome 16 (top) and for chromosome 1 (bottom) for whole blood tissue. 

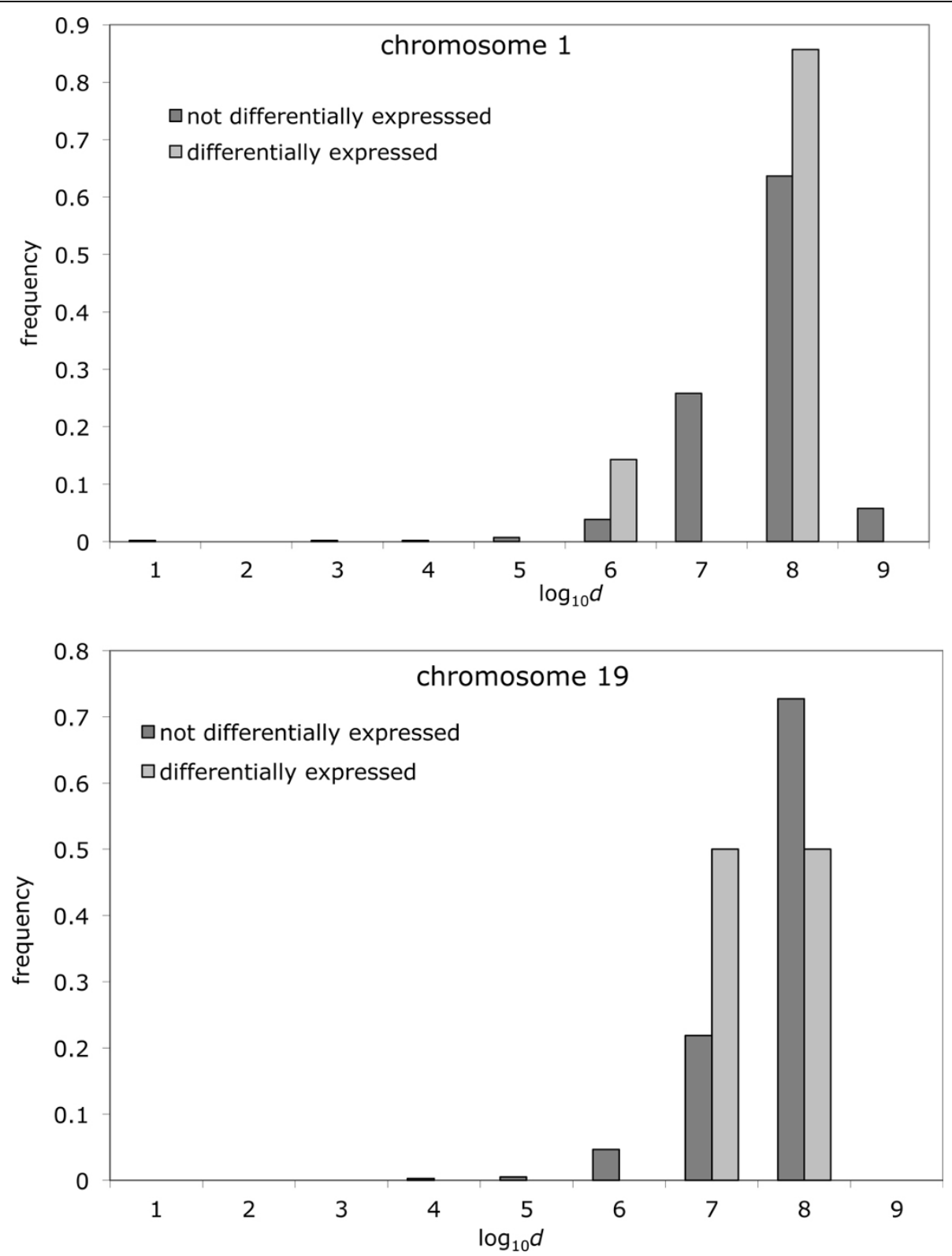

Figure 5 Expression in brain and distance to breakpoint for two chromosomes. Histogram of distances from genes to closest BPR for differentially expressed genes vs. not differentially expressed genes in cerebral cortex tissue for chromosome 1 (top) and for chromosome 19 (bottom).

possible role of the rearrangement process in concert with purifying selection processes, in maintaining or adjusting the function of $R B 1$.

\section{Conclusions}

Genome rearrangement research has been concerned with the creation of breakpoints and their position in the chromosome. The question arises of what the biological consequences of breakpoint creation are, rather than just their structural aspects.
Since a chromosomal rearrangement may occasionally disrupt the spatial connection between a gene and its regulatory regions, we have asked whether proximity to the site of a breakpoint event changes the activity of a gene. We investigated this by comparing the distribution of distances to the nearest breakpoint of genes that change expression after rearrangement with the same distribution for those that do not change. This question has not been investigated previously on a genome-wide basis. 
The data currently available on individual gene expression change across entire genomes for different species is limited. That we found little evidence for rejecting the null hypothesis is attributable to sparse data and to relatively crude measures of fold changes. With the advent of Next Generation Sequencing, quantitative RNA sequence data on many tissues from related species should soon become available. Our computational pipeline may be of utility at that time.

\section{Acknowledgements}

Research funded in part by a Discovery grant from the Natural Sciences and Engineering Research Council of Canada. We thank Christian Baudet for providing technical assistance for running Cassis.

This article has been published as part of BMC Bioinformatics Volume 13 Supplement 3, 2012: ACM Conference on Bioinformatics, Computational Biology and Biomedicine 2011. The full contents of the supplement are available online at http://www.biomedcentral.com/1471-2105/13/S3.

\section{Author details}

${ }^{1} S c h o o l$ of Information Technology \& Engineering, University of Ottawa, Ottawa, K1N 6N5, Canada. ${ }^{2}$ Department of Mathematics \& Statistics, University of Ottawa, Ottawa, K1N 6N5, Canada.

\section{Authors' contributions}

AM and DS formulated the problem. AM designed and implemented this computational pipeline in Java, PostgreSQL 8.4.3 [17] relational database, SQL, R, and MS Excel. AM selected the two gene expression studies, extracted the data sets from the public databases and loaded them into our PostgreSQL database. AM conducted the interpretation of the results. AM and DS contributed equally to the writing of this manuscript.

\section{Competing interests}

The authors declare that they have no competing interests.

Published: 21 March 2012

\section{References}

1. Mitelman F, Johansson B, Mertens F: Mitelman database of chromosome aberrations and gene fusions in cancer. 2010 [http://cgap.nci.nih.gov/ Chromosomes/Mitelman].

2. Nowell PC: Discovery of the Philadelphia chromosome: a personal perspective. J Clin Invest 2007, 117:2033.

3. Sankoff D: The where and wherefore of evolutionary breakpoints. J Biol 2009, 8:66.

4. Marquès-Bonet $\mathrm{T}$, Cáceres $\mathrm{M}$, Bertranpetit J, Preuss TM, Thomas JW, Navarro A: Chromosomal rearrangements and the genomic distribution of gene-expression divergence in humans and chimpanzees. Trends Genet 2004, 20:524-529.

5. Yunis JJ, Prakash O: The origin of man: a pictorial legacy. Science 1982, 215:1525-1530.

6. Lemaitre C, Tannier E, Gautier C, Sagot MF: Precise detection of rearrangement breakpoints in mammalian chromosomes. $B M C$ Bioinformatics 2008, 9:286.

7. Lemaitre C, Zhagloul L, Sagot MF, Gautier C, Arneodo A, Tannier E, Audit B: Analysis of fine-scale mammalian evolutionary breakpoints provides new insight into their relation to genome organisation. BMC Genomics 2009, 10:335.

8. Baudet C, Lemaitre C, Dias Z, Gautier C, Tannier E, Sagot MF: Cassis: detection of rearrangement breakpoints. Bioinformatics 2010, 26:1897-1898.

9. Lu Y, Bar-Joseph Z: Cross species analysis of microarray expression data. Bioinformatics 2009, 25:1476-1483.

10. Dillman JF III, Phillips CS: Comparison of non-human primate and human whole blood tissue gene expression profiles. Toxicol Sci 2005, 87:306-314.

11. Cáceres M, Lachuer J, Zapala MA, Redmond JC, Kudo L, Geschwind DH, Lockhart DJ, Preuss TM, Barlow C: Elevated gene expression levels distinguish human from non-human primate brains. Proc Natl Acad Sci USA 2003, 100:13030-13035.

12. Abyzov A, Gerstein M: AGE: defining breakpoints of genomic structural variants at single-nucleotide resolution, through optimal alignments with gap excision. Bioinformatics 2011, 27:595-603.

13. Trinh P, McLysaght A, Sankoff D: Genomic features in the breakpoint regions between syntenic blocks. Bioinformatics 2004, 20(Suppl 1): i318-i325.

14. BioMart. [http://www.ensembl.org/biomart/martview/]

15. Karolchik D, Baertsch R, Diekhans M, Furey TS, Hinrichs A, Lu YT, Roskin KM, Schwartz M, Sugnet CW, Thomas DJ, Weber RJ, Haussler D, Kent WJ, University of California Santa Cruz: The UCSC Genome Browser Database. Nucleic Acids Res 2003, 31:51-54.

16. Ensembl Genome Browser. [http://www.ensembl.org].

17. PostgreSQL. [http://www.postgresql.org].

18. Laureys G, Speleman F, Opdenakker G, Benoit Y, Leroy J: Constitutional translocation $\mathrm{t}(1 ; 17)(\mathrm{p} 36 ; \mathrm{q} 12-21)$ in a patient with neuroblastoma. Genes Chromosomes Cancer 1990, 2:252-254.

19. Vandepoele K, Van Roy N, Staes K, Speleman F, van Roy F: A novel gene family NBPF: intricate structure generated by gene duplications during primate evolution. Mol Biol Evol 2005, 22:2265-2274.

20. Sudmant PH, Kitzman JO, Antonacci F, Alkan C, Malig M, Tsalenko A, Sampas N, Bruhn L, Shendure J, 1000 Genomes Project, Eichler EE: Diversity of human copy number variation and multicopy genes. Science 2010, 330:641-646.

21. Lee VMY, Goedert M, Trojanowski JQ: Neurodegenerative tauopathies. Annu Rev Neurosci 2001, 24:1121-1159.

22. Berriman J, Serpell LC, Oberg KA, Fink AL, Goedert M, Crowther RA: Tau filaments from human brain and from in vitro assembly of recombinant protein show cross-h structure. Proc Natl Acad Sci USA 2003, 100:9034-9038.

23. Gearing M, Rebeck GW, Hyman BT, Tigges J, Mirra SS: Neuropathology and apolipoprotein E profile of aged chimpanzees: implications for Alzheimer disease. Proc Natl Acad Sci USA 1994, 91:9382-9386.

24. Holzer M, Craxton M, Jakes R, Arendt T, Goeder M: Tau gene (MAPT) sequence variation among primates. Gene 2004, 341:313-322.

25. Sivakumaran TA, Shen P, Wall DP, Do BH, Kucheria K, Oefner PJ: Conservation of the RB1 gene in human and primates. Hum Mutat 2005, 25:396-409.

doi:10.1186/1471-2105-13-S3-S6

Cite this article as: Muñoz and Sankoff: Detection of gene expression changes at chromosomal rearrangement breakpoints in evolution. BMC Bioinformatics 2012 13(Suppl 3):S6.

\section{Submit your next manuscript to BioMed Central and take full advantage of:}

- Convenient online submission

- Thorough peer review

- No space constraints or color figure charges

- Immediate publication on acceptance

- Inclusion in PubMed, CAS, Scopus and Google Scholar

- Research which is freely available for redistribution
Ciomed Central 